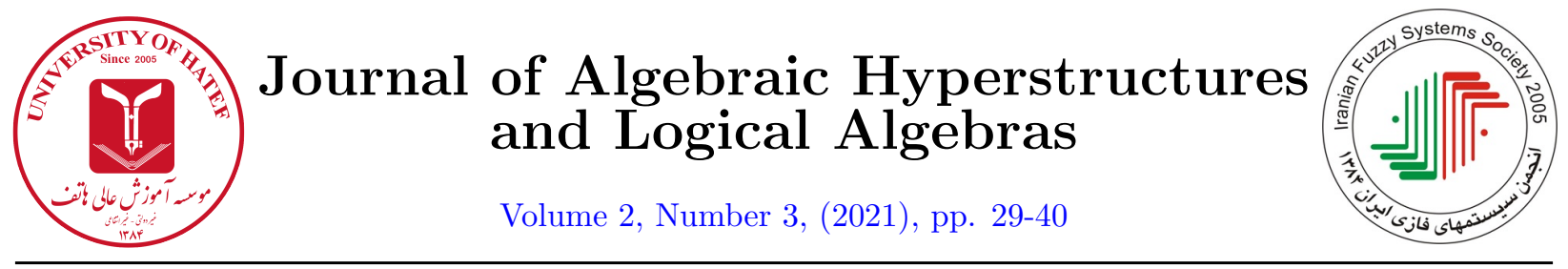

\title{
Implicative soju ideals of BCK-algebras
}

\author{
S.Z. Song ${ }^{1}$ \\ ${ }^{1}$ Department of Mathematics, Jeju National University, Jeju 63243, Korea \\ szsong@jejunu.ac.kr
}

"This paper is dedicated to Professor Young Bae Jun on the occasion of his 70th birthday."

\begin{abstract}
Molodtsov proposed soft set theory to address uncertainty in a parameter manner, and Atanassov introduced intuitionistic fuzzy set which is very useful in providing a flexible model to elaborate uncertainty and vagueness involved in decision making. Using intuitive fuzzy set and soft set, Jun et al. introduced a new notion, "soju structure", as a kind of hybrid structure. The concept of implicative soju ideal in BCK-algebra will be introduced and several properties will be investigated in this article. We will discuss the relationship between soju subalgebra, soju ideal, positive implicative soju ideal, commutative soju ideal and implicative soju ideal. Also, characterizations of implicative soju ideal will be established.
\end{abstract}

\section{Article Information}

Corresponding Author:

S.Z. Song;

Received: March 2021;

Accepted: Invited paper;

Paper type: Original.

\section{Keywords:}

(Positive implicative, commutative) soju ideal, implicative soju ideal.

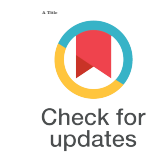

\section{Introduction}

Soft set theory, as the general version of fuzzy set theory, was proposed by Molodtsov [20] to address uncertainty in a parameter manner. Intuitionistic fuzzy set, introduced by Atanassov [4, 5], is also a generalization of fuzzy set theory, and it is very useful in providing a flexible model to elaborate uncertainty and vagueness involved in decision making. Using soft set theory and intuitionistic fuzzy set theory to study

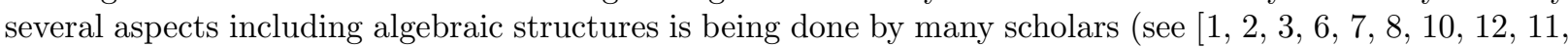
144, 15, [6, [8, [22, [23, [2.5]). Jun et al. used intuitionistic fuzzy set and soft set to introduce a new structure, so called a soju structure, which is a kind of hybrid structure, and then it is applied to BCK/BCI-algebras (see [177, 21, 24]).

In this paper, we introduce the implicative soju ideal in BCK-algebra and investigate several properties. We investigate the relationship between soju subalgebra, soju ideal, positive implicative soju ideal, commutative soju ideal and implicative soju ideal. We provide examples to show that any soju subalgebra (resp., soju ideal, positive implicative soju ideal and commutative soju ideal) is not an implicative soju ideal. We find and present conditions under which soju subalgebra (resp., soju ideal, positive implicative soju ideal and commutative soju ideal) can be implicative soju ideal. We discuss characterizations of implicative soju ideal.

\footnotetext{
https://doi.org/10.52547/HATEF.JAHLA.2.3.3
} 


\section{Preliminaries}

This section lists the already well-known concepts for BCK-algebras and soju structures related to this paper.

\subsection{Basic concepts about BCK-algebras}

A $B C K$-algebra is defined to be an algebra $(X ; *, 0)$ that satisfies the following conditions:

$\left(I_{1}\right)((\tilde{x} * \tilde{y}) *(\tilde{x} * \tilde{z})) *(\tilde{z} * \tilde{y})=0$,

$\left(I_{2}\right)(\tilde{x} *(\tilde{x} * \tilde{y})) * \tilde{y}=0$,

$\left(I_{3}\right) \tilde{x} * \tilde{x}=0$

$\left(I_{4}\right) \tilde{x} * \tilde{y}=0, \tilde{y} * \tilde{x}=0 \Rightarrow \tilde{x}=\tilde{y}$

(K) $0 * \tilde{x}=0$

for all $\tilde{x}, \tilde{y}, \tilde{z} \in X$.

We define an order relation " $\leq$ " on a BCK-algebra $X$ as follows:

$$
(\forall \tilde{x}, \tilde{y} \in X)(\tilde{x} \leq \tilde{y} \Leftrightarrow \tilde{x} * \tilde{y}=0) .
$$

Every BCK-algebra $X$ satisfies:

$$
\begin{aligned}
& (\forall \tilde{x} \in X)(\tilde{x} * 0=\tilde{x}), \\
& (\forall \tilde{x}, \tilde{y}, \tilde{z} \in X)(\tilde{x} \leq \tilde{y} \Rightarrow \tilde{x} * \tilde{z} \leq \tilde{y} * \tilde{z}, \tilde{z} * \tilde{y} \leq \tilde{z} * \tilde{x}), \\
& (\forall \tilde{x}, \tilde{y}, \tilde{z} \in X)((\tilde{x} * \tilde{y}) * \tilde{z}=(\tilde{x} * \tilde{z}) * \tilde{y}) . \\
& (\forall \tilde{x}, \tilde{y}, \tilde{z} \in X)((\tilde{x} * \tilde{z}) *(\tilde{y} * \tilde{z}) \leq \tilde{x} * y) .
\end{aligned}
$$

A BCK-algebra $X$ is said to be

- commutative (see [[T. ]) if $\tilde{x} *(\tilde{x} * \tilde{y})=\tilde{y} *(\tilde{y} * \tilde{x})$ for all $\tilde{x}, \tilde{y} \in X$.

- positive implicative $($ see $[\mathbb{[ \square ]}])$ if $(\tilde{x} * \tilde{z}) *(\tilde{y} * \tilde{z})=(\tilde{x} * \tilde{y}) * \tilde{z}$ for all $\tilde{x}, \tilde{y}, \tilde{z} \in X$.

- implicative (see [[., ] ) if $\tilde{x}=\tilde{x} *(\tilde{y} * \tilde{x})$ for all $\tilde{x}, \tilde{y} \in X$.

A subset $K$ of a BCK-algebra $X$ is called an ideal of $X$ if it satisfies:

$$
\begin{aligned}
& 0 \in K, \\
& (\forall \tilde{x}, \tilde{y} \in X)(\tilde{x} * \tilde{y} \in K, \tilde{y} \in K \Rightarrow \tilde{x} \in K) .
\end{aligned}
$$

A subset $K$ of a BCK-algebra $X$ is called

- a commutative ideal of $X$ (see [[.:] ) if it satisfies ([6) and

$$
(\forall \tilde{x}, \tilde{y}, \tilde{z} \in X)((\tilde{x} * \tilde{y}) * \tilde{z} \in K, \tilde{z} \in K \Rightarrow \tilde{x} *(\tilde{y} *(\tilde{y} * \tilde{x})) \in K) .
$$

- a positive implicative ideal of $X$ (see [[प]]) if it satisfies ([6) and

$$
(\forall \tilde{x}, \tilde{y}, \tilde{z} \in X)((\tilde{x} * \tilde{y}) * \tilde{z} \in K, \tilde{y} * \tilde{z} \in K \Rightarrow \tilde{x} * \tilde{z} \in K) .
$$

- an implicative ideal of $X$ (see [[1.] ) if it satisfies ([6) and

$$
(\forall \tilde{x}, \tilde{y}, \tilde{z} \in X)(((\tilde{x} *(\tilde{y} * x)) * \tilde{z} \in K, \tilde{z} \in K \Rightarrow \tilde{x} \in K) .
$$

For more information on BCK-algebra, please refer to the book [II.] 


\subsection{Basic concepts about soju structures}

In what follows, let $W$ be an initial universe set unless otherwise specified.

Definition 2.1. [I7] Let $X$ be a set of parameters. For any subset $K$ of $X$, let $\lambda:=\left(\varphi_{\lambda}\right.$, $\left.\eta_{\lambda}\right)$ be an intuitionistic fuzzy set in $K$ and $(\tilde{F}, K)$ be a soft set over $W$. Then the pair $(K,\langle\lambda ; \tilde{F}\rangle)$ is called a soju structure related to $K$ (briefly, $K$-soju structure) over $([0,1], W)$. An $X$-soju structure over $([0,1], W)$ is simply called a soju structure over $([0,1], W)$.

Given a soju structure $(X,\langle\lambda ; \tilde{F}\rangle)$ over $([0,1], W)$, we consider the following sets:

$$
\begin{aligned}
& \equiv{ }_{\tilde{F}}^{\lambda}:=\left\{\{x, y\} \subseteq X \mid \varphi_{\lambda}(x)=\varphi_{\lambda}(y), \eta_{\lambda}(x)=\eta_{\lambda}(y), \tilde{F}(x)=\tilde{F}(y)\right\}, \\
& \Omega_{\tilde{F}}^{\lambda}:=\left\{\begin{array}{l|l}
\frac{x}{(y, z)} \in \frac{X}{X \times X} & \begin{array}{l}
\varphi_{\lambda}(x) \geq \min \left\{\varphi_{\lambda}(y), \varphi_{\lambda}(z)\right\} \\
\eta_{\lambda}(x) \leq \max \left\{\eta_{\lambda}(y), \eta_{\lambda}(z)\right\} \\
\tilde{F}(x) \supseteq \tilde{F}(y) \cap \tilde{F}(z)
\end{array}
\end{array}\right\} .
\end{aligned}
$$

It is clear that

$$
\begin{aligned}
& (\forall x, y, z \in X)\left(\frac{x}{(y, z)} \in \Omega_{\tilde{F}}^{\lambda} \Leftrightarrow \frac{x}{(z, y)} \in \Omega_{\tilde{F}}^{\lambda}\right) \\
& (\forall x, y, a, b \in X)\left(\frac{x}{(y, y)} \in \Omega_{\tilde{F}}^{\lambda}, \frac{y}{(a, b)} \in \Omega_{\tilde{F}}^{\lambda} \Rightarrow \frac{x}{(a, b)} \in \Omega_{\tilde{F}}^{\lambda}\right) . \\
& (\forall x, y, z, a \in X)\left(\frac{x}{(y, z)} \in \Omega_{\tilde{F}}^{\lambda}, \frac{y}{(a, a)} \in \Omega_{\tilde{F}}^{\lambda} \Rightarrow \frac{x}{(a, z)} \in \Omega_{\tilde{F}}^{\lambda}\right) . \\
& (\forall x, y, z, a \in X)\left(\frac{x}{(y, z)} \in \Omega_{\tilde{F}}^{\lambda}, \frac{a}{(x, z)} \in \Omega_{\tilde{F}}^{\lambda} \Rightarrow \frac{a}{(y, z)} \in \Omega_{\tilde{F}}^{\lambda}\right) \\
& (\forall x, y, z, a, b \in X)\left(\frac{x}{(y, z)} \in \Omega_{\tilde{F}}^{\lambda}, \frac{y}{(a, b)}, \frac{z}{(a, b)} \in \Omega_{\tilde{F}}^{\lambda} \Rightarrow \frac{x}{(a, b)} \in \Omega_{\tilde{F}}^{\lambda}\right)
\end{aligned}
$$

Definition 2.2. [I7] Let $X$ be a BCK-algebra. A soju structure $(X,\langle\lambda ; \tilde{F}\rangle)$ over $([0,1], W)$ is called a soju subalgebra of $X$ if the following condition is valid.

$$
(\forall x, y \in X)\left(\frac{x * y}{(x, y)} \in \Omega_{\tilde{F}}^{\lambda}\right)
$$

Definition 2.3. [17] Let $X$ be a BCK-algebra. A soju structure $(X,\langle\lambda ; \tilde{F}\rangle)$ over $([0,1], W)$ is called a soju ideal of $X$ if the following conditions are valid.

$$
\begin{aligned}
& (\forall x \in X)\left(\frac{0}{(x, x)} \in \Omega_{\tilde{F}}^{\lambda}\right), \\
& (\forall x, y \in X)\left(\frac{x}{(x * y, y)} \in \Omega_{\tilde{F}}^{\lambda}\right) .
\end{aligned}
$$

Lemma 2.4. [17] Every soju ideal $(X,\langle\lambda ; \tilde{F}\rangle)$ of a BCK-algebra $X$ satisfies:

$$
(\forall x, y \in X)\left(x \leq y \Rightarrow \frac{x}{(y, y)} \in \Omega_{\tilde{F}}^{\lambda}\right)
$$

Lemma 2.5. [17] Every soju ideal $(X,\langle\lambda ; \tilde{F}\rangle)$ of a BCK-algebra $X$ satisfies:

$$
(\forall x, y, z \in X)\left(x * y \leq z \Rightarrow \frac{x}{(y, z)} \in \Omega_{\tilde{F}}^{\lambda}\right)
$$

Definition 2.6. [24] Let $X$ be a BCK-algebra. A soju structure $(X,\langle\lambda ; \tilde{F}\rangle)$ over $([0,1], W)$ is called a positive implicative soju ideal of $X$ if it satisfies (एप) and

$$
(\forall x, y, z \in X)\left(\frac{x * z}{((x * y) * z, y * z)} \in \Omega_{\tilde{F}}^{\lambda}\right)
$$


Definition 2.7. [21] Let $X$ be a BCK-algebra. A soju structure $(X,\langle\lambda ; \tilde{F}\rangle)$ over $([0,1], W)$ is called a

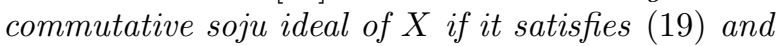

$$
(\forall x, y, z \in X)\left(\frac{x *(y *(y * x))}{((x * y) * z, z)} \in \Omega_{\tilde{F}}^{\lambda}\right) .
$$

Recall that every positive implicative and commutative soju ideal is a soju ideal (see [2I, [24]).

Lemma 2.8. [2]] Given a soju ideal $(X,\langle\lambda ; \tilde{F}\rangle)$ of a BCK-algebra $X$, the following are equivalent.

(i) $(X,\langle\lambda ; \tilde{F}\rangle)$ is a commutative soju ideal of $X$.

(ii) $(X,\langle\lambda ; \tilde{F}\rangle)$ satisfies:

$$
(\forall x, y \in X)\left(\frac{x *(y *(y * x))}{(x * y, x * y)} \in \Omega_{\tilde{F}}^{\lambda}\right) .
$$

\section{Implicative soju ideals of BCK-algebras}

In this section, we define an implicative soju ideal in a BCK-algebra, and investigate several properties. The symbol $X$ in this section represent a BCK-algebra unless otherwise specified.

Definition 3.1. A soju structure $(X,\langle\lambda ; \tilde{F}\rangle)$ over $([0,1], W)$ is called an implicative soju ideal of $X$ if it satisfies (ㅍ) and

$$
(\forall x, y, z \in X)\left(\frac{x}{((x *(y * x)) * z, z)} \in \Omega_{\tilde{F}}^{\lambda}\right) .
$$

Example 3.2. (1) Let $X=\{0,1,2,3\}$ be a set with the Cayley table which is given in Table $\square$.

Table 1: Cayley table for the binary operation "*”

\begin{tabular}{l|llll}
\hline$*$ & 0 & 1 & 2 & 3 \\
\hline 0 & 0 & 0 & 0 & 0 \\
1 & 1 & 0 & 1 & 1 \\
2 & 2 & 2 & 0 & 2 \\
3 & 3 & 3 & 3 & 0 \\
\hline
\end{tabular}

Then $X$ is a BCK-algebra (see [ए耳] ). Let $(X,\langle\lambda ; \tilde{F}\rangle)$ be a soju structure over $([0,1], W)$ which is defined by Table 圆,

Table 2: Tabular representation of $(X,\langle\lambda ; \tilde{F}\rangle)$

\begin{tabular}{c|ccc}
\hline$X$ & $\varphi_{\lambda}(x)$ & $\eta_{\lambda}(x)$ & $\tilde{F}(x)$ \\
\hline 0 & 0.77 & 0.21 & $\beta_{1}$ \\
1 & 0.58 & 0.39 & $\beta_{3}$ \\
2 & 0.33 & 0.54 & $\beta_{2}$ \\
3 & 0.45 & 0.32 & $\beta_{4}$ \\
\hline
\end{tabular}

where $\beta_{1} \supsetneq \beta_{2} \supsetneq \beta_{3} \supsetneq \beta_{4} \neq \emptyset$ in $2^{W}$. It is routine to verify that $(X,\langle\lambda ; \tilde{F}\rangle)$ is an implicative soju ideal of $X$.

(2) Given an implicative ideal $K$ of $X$, define a soju structure $(X,\langle\lambda ; \tilde{F}\rangle)$ over $([0,1], W)$ as follows:

$$
\begin{aligned}
& \lambda:=\left(\varphi_{\lambda}, \eta_{\lambda}\right): X \rightarrow[0,1] \times[0,1], x \mapsto \begin{cases}(t, s) & \text { if } x \in K, \\
(0,1) & \text { otherwise, },\end{cases} \\
& \tilde{F}: X \rightarrow 2^{W}, x \mapsto \begin{cases}\alpha & \text { if } x \in K, \\
\beta & \text { otherwise, }\end{cases}
\end{aligned}
$$


where $(t, s) \in(0,1] \times[0,1)$ and $\alpha, \beta \in 2^{W}$ with $t+s \leq 1$ and $\alpha \supsetneq \beta$. Then $(X,\langle\lambda ; \tilde{F}\rangle)$ is an implicative soju ideal of $X$.

We set up the relationship between soju ideal and implicative soju ideal.

Theorem 3.3. Every implicative soju ideal is a soju ideal.

Proof. Let $(X,\langle\lambda ; \tilde{F}\rangle)$ be an implicative soju ideal of $X$. If we take $y=x$ and $z=y$ in (26) and use $\left(I_{3}\right)$

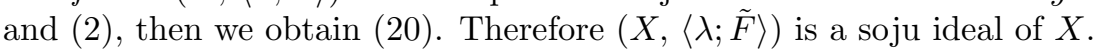

Corollary 3.4. Every implicative soju ideal is a soju subalgebra.

Proof. Straightforward.

The example below shows that there exist a soju ideal and a soju subalgebra which is not an implicative soju ideal.

Example 3.5. Let $X=\{0,1,2,3,4\}$ be a set with the binary operation "*" which is given in Table 圆. Then

Table 3: Cayley table for the binary operation "*"

\begin{tabular}{l|lllll}
\hline$*$ & 0 & 1 & 2 & 3 & 4 \\
\hline 0 & 0 & 0 & 0 & 0 & 0 \\
1 & 1 & 0 & 1 & 0 & 0 \\
2 & 2 & 2 & 0 & 0 & 0 \\
3 & 3 & 3 & 3 & 0 & 0 \\
4 & 4 & 3 & 4 & 1 & 0 \\
\hline
\end{tabular}

$(X, *, 0)$ is a BCK-algebra (see $[\mathbb{[ 1 .}])$. Let $(X,\langle\lambda ; \tilde{F}\rangle)$ be a soju structure over $([0,1], W)$, where $W=\mathbb{Z}$, which is defined by Table $\mathbf{q}$. It is routine to check that $(X,\langle\lambda ; \tilde{F}\rangle)$ is a soju ideal, and hence a soju subalgebra

Table 4: Tabular representation of $(X,\langle\lambda ; \tilde{F}\rangle)$

\begin{tabular}{c|ccc}
\hline$X$ & $\varphi_{\lambda}(x)$ & $\eta_{\lambda}(x)$ & $\tilde{F}(x)$ \\
\hline 0 & 0.65 & 0.29 & $\mathbb{N}$ \\
1 & 0.57 & 0.42 & $2 \mathbb{N}$ \\
2 & 0.43 & 0.32 & $2 \mathbb{N}$ \\
3 & 0.34 & 0.54 & $4 \mathbb{N}$ \\
4 & 0.34 & 0.54 & $4 \mathbb{N}$ \\
\hline
\end{tabular}

of $X$. But it is not an implicative soju ideal of $X$ since $\frac{1}{((1 *(3 * 1)) * 2,2)} \notin \Omega_{\tilde{F}}^{\lambda}$.

It is natural to ask under what conditions any soju ideal or any soju subalgebra can be an implicative soju ideal. Below we will provide those conditions.

Lemma 3.6. Every soju ideal $(X,\langle\lambda ; \tilde{F}\rangle)$ of $X$ satisfies:

$$
(\forall x, y \in X)\left(\frac{x}{(y, 0)} \in \Omega_{\tilde{F}}^{\lambda} \Leftrightarrow \frac{x}{(y, y)} \in \Omega_{\tilde{F}}^{\lambda}\right) .
$$

Proof. Straightforward.

Theorem 3.7. Given a soju ideal $(X,\langle\lambda ; \tilde{F}\rangle)$ of $X$, the following are equivalent.

(i) $(X,\langle\lambda ; \tilde{F}\rangle)$ is an implicative soju ideal of $X$. 
(ii) $(X,\langle\lambda ; \tilde{F}\rangle)$ satisfies:

$$
(\forall x, y \in X)\left(\frac{x}{(x *(y * x), x *(y * x))} \in \Omega_{\tilde{F}}^{\lambda}\right) .
$$

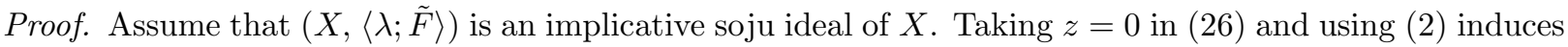
$\frac{x}{(x *(y * x), 0)}=\frac{x}{((x *(y * x)) * 0,0)} \in \Omega_{\tilde{F}}^{\lambda}$ for all $x, y \in X$. It follows from Lemma 3.6 that $\frac{x}{(x *(y * x), x *(y * x))} \in \Omega_{\tilde{F}}^{\lambda}$ for all $x, y \in X$.

Conversely, suppose $(X,\langle\lambda ; \tilde{F}\rangle)$ satisfies (更). Since $(X,\langle\lambda ; \tilde{F}\rangle)$ of $X$ is a soju ideal of $X$, we have $\frac{x *(y * x)}{((x *(y * x)) * z, z)} \in \Omega_{\tilde{F}}^{\lambda}$ for all $x, y, z \in X$. It follows from (四) and (피) that $\frac{x}{((x *(y * x)) * z, z)} \in \Omega_{\tilde{F}}^{\lambda}$ for all $x, y, z \in X$. Therefore $(X,\langle\lambda ; \tilde{F}\rangle)$ is an implicative soju ideal of $X$.

Lemma 3.8. Every soju ideal $(X,\langle\lambda ; \tilde{F}\rangle)$ of $X$ satisfies:

$$
\begin{aligned}
& (\forall x, y \in X)\left(\frac{x}{(y, y)} \in \Omega_{\tilde{F}}^{\lambda}, \frac{y}{(x, x)} \in \Omega_{\tilde{F}}^{\lambda} \Rightarrow\{x, y\} \in \equiv_{\tilde{F}}^{\lambda}\right) . \\
& (\forall x, y \in X)\left(\frac{x}{(x *(y * x), x *(y * x))} \in \Omega_{\tilde{F}}^{\lambda} \Rightarrow\{x, x *(y * x)\} \in \equiv_{\tilde{F}}^{\lambda}\right) . \\
& (\forall x, y \in X)\left(\{x, y\} \in \equiv_{\tilde{F}}^{\lambda}, \frac{y}{(a, b)} \in \Omega_{\tilde{F}}^{\lambda} \Rightarrow \frac{x}{(a, b)} \in \Omega_{\tilde{F}}^{\lambda}\right) .
\end{aligned}
$$

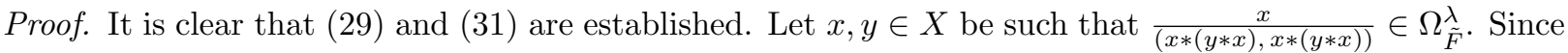
$x *(y * x) \leq x$, it follows from Lemma ए.4 that $\frac{x *(y * x)}{(x, x)} \in \Omega_{\tilde{F}}^{\lambda}$ for all $x, y \in X$. Hence $\{x, x *(y * x)\} \in \equiv_{\tilde{F}}^{\lambda}$ by $($ (2Q1) $)$.

Theorem 3.9. Given a soju subalgebra $(X,\langle\lambda ; \tilde{F}\rangle)$ of $X$, the following are equivalent.

(i) $(X,\langle\lambda ; \tilde{F}\rangle)$ is an implicative soju ideal of $X$.

(ii) $(X,\langle\lambda ; \tilde{F}\rangle)$ satisfies:

$$
(\forall x, y, z, a \in X)\left((x *(y * x)) * z \leq a \Rightarrow \frac{x}{(z, a)} \in \Omega_{\tilde{F}}^{\lambda}\right) .
$$

Proof. Let $(X,\langle\lambda ; \tilde{F}\rangle)$ be an implicative soju ideal of $X$. Then it is a soju ideal of $X$ (see Theorem 5.3 ). If we combine (ㅍ) with (Bत), then $\{x, x *(y * x)\} \in \equiv_{\tilde{F}}^{\lambda}$. Let $x, y, z, a \in X$ be such that $(x *(y * x)) * z \leq a$. Then $\frac{x *(y * x)}{(z, a)} \in \Omega_{\tilde{F}}^{\lambda}$ by Lemma W. Hence $\frac{x}{(z, a)} \in \Omega_{\tilde{F}}^{\lambda}$ by $(\boldsymbol{\Xi Z})$.

Conversely, suppose that $(X,\langle\lambda ; \tilde{F}\rangle)$ satisfies (B2)). Since $(X,\langle\lambda ; \tilde{F}\rangle)$ is a soju subalgebra of $X$, we have $\frac{0}{(x, x)}=\frac{x * x}{(x, x)} \in \Omega_{\tilde{F}}^{\lambda}$ for all $x \in X$. Since $(x *(y * x)) *((x *(y * x)) * z) \leq z$, we get $\frac{x}{((x *(y * x)) * z, z)} \in \Omega_{\tilde{F}}^{\lambda}$ for all $x, y, z \in X$ by (32). Consequently, $(X,\langle\lambda ; \tilde{F}\rangle)$ is an implicative soju ideal of $X$.

Theorem 3.10. In an implicative BCK-algebra, every soju ideal is an implicative soju ideal.

Proof. Let $(X,\langle\lambda ; \tilde{F}\rangle)$ be a soju ideal of an implicative BCK-algebra $X$. Since $X$ is an impliative BCKalgebra, we get $x=x *(y * x)$ for all $x, y \in X$. Hence

$$
(x *((x *(y * x)) * z)) * z=(x * z) *((x *(y * x)) * z)=((x *(y * x)) * z) *((x *(y * x)) * z)=0,
$$

that is, $x *((x *(y * x)) * z) \leq z$ for all $x, y, z \in X$. It follows from Lemma $\left[2.5\right.$ that $\frac{x}{((x *(y * x)) * z, z)} \in \Omega_{\tilde{F}}^{\lambda}$ for all $x, y, z \in X$. Therefore $(X,\langle\lambda ; \tilde{F}\rangle)$ is an implicative soju ideal of $X$.

Corollary 3.11. If every BCK-algebra $X$ satisfies either of the following:

(i) $(\forall x, y \in X)((x *(x * y)) *(x * y)=y *(y * x))$,

(ii) $(\forall x, y \in X)((x *(x * y)) *(y * x)=y *(y * x))$,

(iii) $(\forall x, y \in X)((x *(x * y)) *(x * y)=(y *(y * x)) *(y * x))$, 
then every soju ideal is an implicative soju ideal.

The following example shows that even if $X$ is an implicative BCK-algebra, any soju subalgebra of $X$ may not be an implicative soju ideal of $X$.

Example 3.12. Let $X=\{0,1,2,3,4\}$ be a set with the binary operation "*" which is given in Table 囯.

Table 5: Cayley table for the binary operation "*”

\begin{tabular}{l|lllll}
\hline$*$ & 0 & 1 & 2 & 3 & 4 \\
\hline 0 & 0 & 0 & 0 & 0 & 0 \\
1 & 1 & 0 & 1 & 0 & 1 \\
2 & 2 & 2 & 0 & 0 & 2 \\
3 & 3 & 2 & 1 & 0 & 3 \\
4 & 4 & 4 & 4 & 4 & 0 \\
\hline
\end{tabular}

Then $(X, *, 0)$ is an implicative BCK-algebra (see [[प] $)$. Let $(X,\langle\lambda ; \tilde{F}\rangle)$ be a soju structure over $([0,1], W)$, where $W=\mathbb{Z}$, which is defined by Table $\mathbb{6}$. It is routine to verify that $(X,\langle\lambda ; \tilde{F}\rangle)$ is a soju subalgebra of $X$.

Table 6: Tabular representation of $(X,\langle\lambda ; \tilde{F}\rangle)$

\begin{tabular}{c|ccc}
\hline$X$ & $\varphi_{\lambda}(x)$ & $\eta_{\lambda}(x)$ & $\tilde{F}(x)$ \\
\hline 0 & 0.63 & 0.19 & $2 \mathbb{Z}$ \\
1 & 0.56 & 0.28 & $8 \mathbb{N}$ \\
2 & 0.47 & 0.32 & $8 \mathbb{N}$ \\
3 & 0.47 & 0.51 & $4 \mathbb{Z}$ \\
4 & 0.33 & 0.51 & $4 \mathbb{N}$ \\
\hline
\end{tabular}

But it is not an implicative soju ideal of $X$ since $\frac{3}{((3 *(2 * 3)) * 1,1)} \notin \Omega_{\tilde{F}}^{\lambda}$.

We build the relationship between positive implicative soju ideal and implicative soju ideal.

Theorem 3.13. Every implicative soju ideal is a positive implicative soju ideal.

Proof. Let $(X,\langle\lambda ; \tilde{F}\rangle)$ be an implicative soju ideal of $X$. Then $(X,\langle\lambda ; \tilde{F}\rangle)$ is a soju ideal of $X$ (see Theorem B.3). Let $x, y, z \in X$. Since $((x * z) * z) *(y * z) \leq(x * z) * y=(x * y) * z$ and

$$
(x * z) *(x *(x * z))=(x *(x *(x * z))) * z=(x * z) * z,
$$

it follows from Lemma 2.5 that

$$
\frac{(x * z) *(x *(x * z))}{(y * z,(x * y) * z)}=\frac{(x * z) * z}{(y * z,(x * y) * z)} \in \Omega_{\tilde{F}}^{\lambda} .
$$

Note that $\frac{x * z}{((x * z) *(x *(x * z)),(x * z) *(x *(x * z)))} \in \Omega_{\tilde{F}}^{\lambda}$ by Theorem [3.], and hence $\{x * z,(x * z) *(x *(x * z))\} \in \equiv_{\tilde{F}}^{\lambda}$ by (而). Thus $\frac{x * z}{(y * z,(x * y) * z)} \in \Omega_{\tilde{F}}^{\lambda}$ by (BU) , and therefore $(X,\langle\lambda ; \tilde{F}\rangle)$ is a positive implicative soju ideal of $X$.

The following example shows that the converse of Theorem $[]$.3 is not ture in general.

Example 3.14. Let $X=\{0,1,2,3,4\}$ be a set with the binary operation "*" which is given in Table $\square$. Then $(X, *, 0)$ is a BCK-algebra (see [[प] $)$. Let $(X,\langle\lambda ; \tilde{F}\rangle)$ be a soju structure over $([0,1], W)$, where $W=\mathbb{Z}$, which is defined by Table $\mathbf{\square}$.

It is routine to verify that $(X,\langle\lambda ; \tilde{F}\rangle)$ is a positive implicative soju ideal of $X$. But it is not an implicative soju ideal of $X$ since $\frac{2}{((2 *(4 * 2)) * 3,3)} \notin \Omega_{\tilde{F}}^{\lambda}$. 
Table 7: Cayley table for the binary operation "*”

\begin{tabular}{l|lllll}
\hline$*$ & 0 & 1 & 2 & 3 & 4 \\
\hline 0 & 0 & 0 & 0 & 0 & 0 \\
1 & 1 & 0 & 0 & 1 & 0 \\
2 & 2 & 2 & 0 & 2 & 0 \\
3 & 3 & 3 & 3 & 0 & 3 \\
4 & 4 & 4 & 4 & 4 & 0 \\
\hline
\end{tabular}

Table 8: Tabular representation of $(X,\langle\lambda ; \tilde{F}\rangle)$

\begin{tabular}{c|ccc}
\hline$X$ & $\varphi_{\lambda}(x)$ & $\eta_{\lambda}(x)$ & $\tilde{F}(x)$ \\
\hline 0 & 0.66 & 0.09 & $2 \mathbb{Z}$ \\
1 & 0.54 & 0.35 & $4 \mathbb{Z}$ \\
2 & 0.42 & 0.42 & $4 \mathbb{N}$ \\
3 & 0.49 & 0.23 & $16 \mathbb{N}$ \\
4 & 0.34 & 0.52 & $8 \mathbb{N}$ \\
\hline
\end{tabular}

Proposition 3.15. Every implicative soju ideal $(X,\langle\lambda ; \tilde{F}\rangle)$ of $X$ satisfies:

$$
(\forall x, y \in X)\left(\frac{x *(x * y)}{(y *(y * x), y *(y * x))} \in \Omega_{\tilde{F}}^{\lambda}\right) .
$$

Proof. Let $x, y \in X$. Since

$$
(x *(x * y)) *(y *(x *(x * y))) \leq(x *(x * y)) *(y * x)=(x *(y * x)) *(x * y) \leq y *(y * x),
$$

it follows from (更) and Lemma 2.4 that

$$
\frac{((x *(x * y)) *(y *(x *(x * y)))) * 0}{(y *(y * x), y *(y * x))}=\frac{(x *(x * y)) *(y *(x *(x * y)))}{(y *(y * x), y *(y * x))} \in \Omega_{\tilde{F}}^{\lambda} .
$$

Using (피) leads to $\frac{x *(x * y)}{(((x *(x * y)) *(y *(x *(x * y)))) * 0,0)} \in \Omega_{\tilde{F}}^{\lambda}$, and it is equivalent to

$$
\frac{x *(x * y)}{(((x *(x * y)) *(y *(x *(x * y)))) * 0,((x *(x * y)) *(y *(x *(x * y)))) * 0)} \in \Omega_{\tilde{F}}^{\lambda}
$$

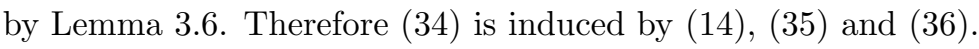

We concider conditions for a positive implicative soju ideal to be an implicative soju ideal.

Lemma 3.16. [24] Let $(X,\langle\lambda ; \tilde{F}\rangle)$ be a soju ideal of $X$. Then it is a positive implicative soju ideal of $X$ if and only if it satisfies:

$$
(\forall x, y \in X)\left(\frac{x * y}{((x * y) * y,(x * y) * y)} \in \Omega_{\tilde{F}}^{\lambda}\right) .
$$

Theorem 3.17. If every positive implicative soju ideal $(X,\langle\lambda ; \tilde{F}\rangle)$ of $X$ satisfies the condition (Bय), then it is an implicative soju ideal of $X$.

Proof. Let $(X,\langle\lambda ; \tilde{F}\rangle)$ be a positive implicative soju ideal of $X$ that satisfies the condition (34). Then $(X$, $\langle\lambda ; \tilde{F}\rangle)$ is a soju ideal of $X$. Hence

$$
\frac{x *(y * x)}{((x *(y * x)) * z, z)} \in \Omega_{\tilde{F}}^{\lambda}
$$

for all $x, y, z \in X$. Since $(y *(y * x)) *(y * x) \leq x *(y * x)$, we get

$$
\frac{(y *(y * x)) *(y * x)}{(x *(y * x), x *(y * x))} \in \Omega_{\tilde{F}}^{\lambda}
$$


by Lemma [2.4. Using Lemma [3.T6 gives

$$
\frac{y *(y * x)}{((y *(y * x)) *(y * x),(y *(y * x)) *(y * x))} \in \Omega_{\tilde{F}}^{\lambda},
$$

and so

$$
\frac{x *(x * y)}{((y *(y * x)) *(y * x),(y *(y * x)) *(y * x))} \in \Omega_{\tilde{F}}^{\lambda}
$$

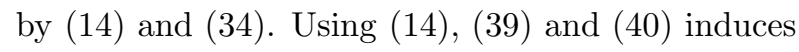

$$
\frac{y *(y * x)}{(x *(y * x), x *(y * x))} \in \Omega_{\tilde{F}}^{\lambda} .
$$

Also, if we use (피), (34) and (42), then we get

$$
\frac{x *(x * y)}{(x *(y * x), x *(y * x))} \in \Omega_{\tilde{F}}^{\lambda} .
$$

Since $(x * y) * z \leq x * y \leq x *(y * x)$, it follows from Lemma 2.4 that

$$
\frac{(x * y) * z}{(x *(y * x), x *(y * x))} \in \Omega_{\tilde{F}}^{\lambda} .
$$

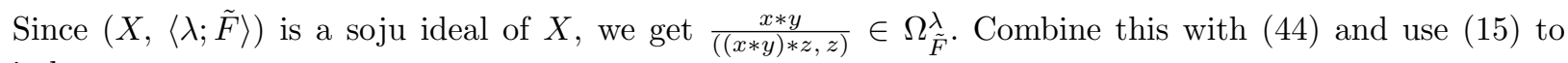
induce

$$
\frac{x * y}{(x *(y * x), z)} \in \Omega_{\tilde{F}}^{\lambda}
$$

Also, combine ([38) with (105) and use ([16) to induce

$$
\frac{x * y}{((x *(y * x)) * z, z)} \in \Omega_{\tilde{F}}^{\lambda} .
$$

Since $\frac{x}{(x *(x * y), x * y)} \in \Omega_{\tilde{F}}^{\lambda}$, it follows from (미) and (미) that

$$
\frac{x}{((y *(y * x)) *(y * x), x * y)} \in \Omega_{\tilde{F}}^{\lambda} .
$$

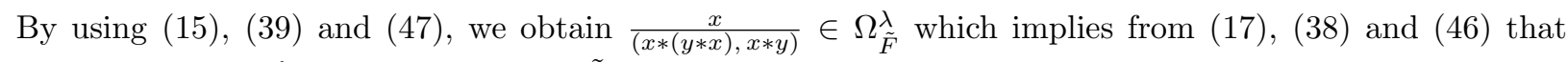
$\frac{x}{((x *(y * x)) * z, z)} \in \Omega_{\tilde{F}}^{\lambda}$. Therefore $(X,\langle\lambda ; \tilde{F}\rangle)$ is an implicative soju ideal of $X$.

We look into the relationship between commutative soju ideal and implicative soju ideal.

Lemma 3.18. [2I] Given a soju ideal $(X,\langle\lambda ; \tilde{F}\rangle)$ of $X$, the following are equivalent.

(i) $(X,\langle\lambda ; \tilde{F}\rangle)$ is a commutative soju ideal of $X$.

(ii) $(X,\langle\lambda ; \tilde{F}\rangle)$ satisfies:

$$
(\forall x, y \in X)\left(\frac{x *(y *(y * x))}{(x * y, x * y)} \in \Omega_{\tilde{F}}^{\lambda}\right)
$$

Theorem 3.19. Every implicative soju ideal is a commutative soju ideal.

Proof. Let $(X,\langle\lambda ; \tilde{F}\rangle)$ be an implicative soju ideal of $X$. Then $(X,\langle\lambda ; \tilde{F}\rangle)$ is a soju ideal of $X$ (see Theorem [3.3). For every $x, y \in X$, we have

$$
\begin{aligned}
& (x *(y *(y * x))) *(y *(x *(y *(y * x)))) \leq(x *(y *(y * x))) *(y * x) \\
& =(x *(y * x)) *(y *(y * x)) \leq x * y
\end{aligned}
$$

which implies from Lemma [2] that $\frac{(x *(y *(y * x))) *(y *(x *(y *(y * x))))}{(x * y, x * y)} \in \Omega_{\tilde{F}}^{\lambda}$. It follows from ([4]) and Theorem

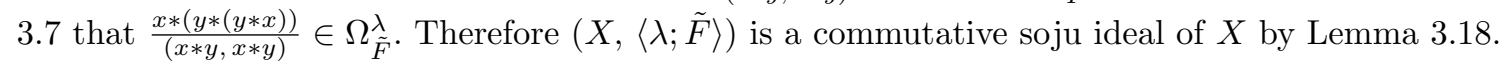


Table 9: Cayley table for the binary operation "*”

\begin{tabular}{l|llll}
\hline$*$ & 0 & 1 & 2 & 3 \\
\hline 0 & 0 & 0 & 0 & 0 \\
1 & 1 & 0 & 0 & 1 \\
2 & 2 & 1 & 0 & 2 \\
3 & 3 & 3 & 3 & 0 \\
\hline
\end{tabular}

Table 10: Tabular representation of $(X,\langle\lambda ; \tilde{F}\rangle)$

\begin{tabular}{c|ccc}
\hline$X$ & $\varphi_{\lambda}(x)$ & $\eta_{\lambda}(x)$ & $\tilde{F}(x)$ \\
\hline 0 & 0.77 & 0.21 & $\beta_{1}$ \\
1 & 0.52 & 0.41 & $\beta_{3}$ \\
2 & 0.52 & 0.41 & $\beta_{3}$ \\
3 & 0.43 & 0.34 & $\beta_{2}$ \\
\hline
\end{tabular}

The next example shows that the converse of Theorem $\$ 1.19$ is not ture in general.

Example 3.20. Let $X=\{0,1,2,3\}$ be a set with the Cayley table which is given in Table $⿴$.

Then $X$ is a BCK-algebra (see [1].9]). Let $(X,\langle\lambda ; \tilde{F}\rangle)$ be a soju structure over $([0,1], W)$ which is defined by Table $[0$

where $\beta_{1} \supsetneq \beta_{2} \supsetneq \beta_{3} \supsetneq \beta_{4} \neq \emptyset$ in $2^{W}$. It is routine to verify that $(X,\langle\lambda ; \tilde{F}\rangle)$ is a commutative soju ideal, and hence a soju ideal, of $X$. Since $\frac{1}{(1 *(2 * 1), 1 *(2 * 1))}=\frac{1}{(0,0)} \notin \Omega_{\tilde{F}}^{\lambda}$, we know that $(X,\langle\lambda ; \tilde{F}\rangle)$ is not an implicative soju ideal of $X$ by Theorem [3.7.

We provide conditions for a commutative soju ideal to be an implicative soju ideal.

Lemma 3.21. If a soju ideal $(X,\langle\lambda ; \tilde{F}\rangle)$ of $X$ satisfies the condition (37), then

$$
(\forall x, y \in X)\left((x * y,(x * y) * y) \in \equiv_{\tilde{F}}^{\lambda}\right) .
$$

Proof. Assume that a soju ideal $(X,\langle\lambda ; \tilde{F}\rangle)$ of $X$ satisfies the condition (B7) and let $x, y \in X$. Since $(x * y) * y \leq x * y$, it follows from Lemma ए. that $\frac{(x * y) * y}{(x * y, x * y)} \in \Omega_{\tilde{F}}^{\lambda}$. If we combine this with (B7), then $(x * y,(x * y) * y) \in \equiv \underset{\tilde{F}}{\lambda}$.

Corollary 3.22. Every positive implicative soju ideal $(X,\langle\lambda ; \tilde{F}\rangle)$ of $X$ satisfies the condition (4.9)).

Lemma 3.23. If a soju ideal $(X,\langle\lambda ; \tilde{F}\rangle)$ of $X$ satisfies the condition (2.5), then

$$
(\forall x, y \in X)\left(\left(x * y, x *(y *(y * x)) \in \equiv_{\tilde{F}}^{\lambda}\right) .\right.
$$

Proof. Let $(X,\langle\lambda ; \tilde{F}\rangle)$ be a soju ideal of $X$ satisfies the condition (匹5). Since $x * y \leq x *(y *(y * x))$, we get $\frac{x * y}{(x *(y *(y * x)), x *(y *(y * x)))} \in \Omega_{\tilde{F}}^{\lambda}$ by Lemma ए.4. If we combine this with (匹.]), then we obtain $(x * y, x *(y *$ $(y * x)) \in \equiv_{\tilde{F}}^{\lambda}$ for all $x, y \in X$.

Corollary 3.24. Every commutative soju ideal $(X,\langle\lambda ; \tilde{F}\rangle)$ of $X$ satisfies the condition (501).

Theorem 3.25. If a commutative soju ideal $(X,\langle\lambda ; \tilde{F}\rangle)$ of $X$ satisfies the condition (B7), then it is an implicative soju ideal of $X$.

Proof. Let $(X,\langle\lambda ; \tilde{F}\rangle)$ be a commutative soju ideal of $X$ that satisfies the condition (Bच). Then $(X,\langle\lambda ; \tilde{F}\rangle)$ is a soju ideal of $X$. Let $x, y \in X$. Since $(y *(y * x)) *(y * x) \leq x *(y * x)$, it follows from Lemma [.4 that

$$
\frac{(y *(y * x)) *(y * x)}{(x *(y * x), x *(y * x))} \in \Omega_{\tilde{F}}^{\lambda} .
$$


By the condition (37), we know that $\frac{y *(y * x)}{((y *(y * x)) *(y * x),(y *(y * x)) *(y * x))} \in \Omega_{\widetilde{F}}^{\lambda}$ and so

$$
(y *(y * x),(y *(y * x)) *(y * x)) \in \stackrel{ }{\tilde{F}}^{\lambda}
$$

by Lemma 3.27 . Using (四), (5ل) and (52) leads to

$$
\frac{y *(y * x)}{(x *(y * x), x *(y * x))} \in \Omega_{\tilde{F}}^{\lambda} .
$$

On the other hand, since $x * y \leq x *(y * x)$, we get $\frac{x * y}{(x *(y * x), x *(y * x))} \in \Omega_{\widetilde{F}}^{\lambda}$, and so

$$
\frac{x *(y *(y * x))}{(x *(y * x), x *(y * x))} \in \Omega_{\tilde{F}}^{\lambda}
$$

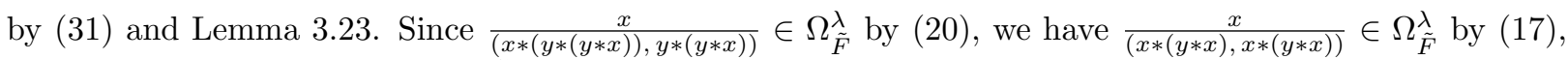
(5.3) and (54). Therefore $(X,\langle\lambda ; \tilde{F}\rangle)$ is an implicative soju ideal of $X$ by Theorem $[.7$.

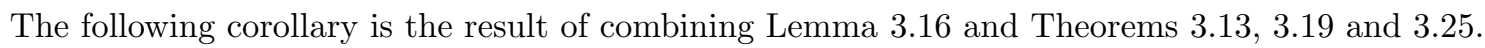

Corollary 3.26. A soju structure $(X,\langle\lambda ; \tilde{F}\rangle)$ over $([0,1], W)$ is an implicative soju ideal of $X$ if and only if it is both a commutative soju ideal and a positive implicative soju ideal of $X$.

\section{Conclusion}

We introduced the concept of implicative soju ideal in BCK-algebra and further explored various properties. In consideration of $\mathrm{A}$ and $\mathrm{B}$, which are described next:

(1) $\left\{\begin{array}{l}\text { A: Soju subalgebra } \\ \text { B: Implicative soju ideal }\end{array}\right.$

(2) $\left\{\begin{array}{l}\text { A: Soju ideal } \\ \text { B: Implicative soju ideal }\end{array}\right.$

(3) $\left\{\begin{array}{l}\text { A: Positive implicative soju ideal } \\ \text { B: Implicative soju ideal }\end{array}\right.$

(4) $\left\{\begin{array}{l}\text { A: Commutative soju ideal } \\ \text { B: Implicative soju ideal, }\end{array}\right.$

we looked into the relationship between A and B. We proved that B becomes A, and gave examples of A not being B. We found and presented conditions under which A can be B. Our future work will utilize ideas and results in this paper to study the substructures of algebraic systems related to BCK/BCI algebra.

\section{Acknowledgment}

This research was supported by Basic Science Research Program through the National Research Foundation of Korea (NRF) funded by the Ministry of Education (NRF-2016R1D1A1B02006812).

\section{References}

[1] U. Acar, F. Koyuncu, B. Tanay, Soft sets and soft rings, Computers and Mathematics with Applications, 59 (2010), 3458-3463.

[2] M. Agarwal, K.K. Biswasa, M. Hanmandlu, Generalized intuitionistic fuzzy soft sets with applications in decision-making, Applied Soft Computing, 13 (2013), 3552-3566.

[3] H. Aktas, N. Cagman, Soft sets and soft groups, Information Sciences, 177 (2007), 2726-2735. 
[4] K.T. Atanassov, Intuitionistic fuzzy sets, Fuzzy Sets and Systems, 20(1) (1986), 87-96.

[5] K.T. Atanassov, New operations defined over the intuitionistic fuzzy sets, Fuzzy Sets and Systems, 61(2) (1994), 137-142.

[6] Z. Bashir, J. Wạtróbski, T. Rashid, W. Salabun, J. Ali, Intuitionistic-fuzzy goals in Zero-Sum multi criteria matrix games, Symmetry, 9 (2017), 158.

[7] S.K. De, R. Biswas, A.R. Roy, An application of intuitionistic fuzzy sets in medical diagnosis, Fuzzy Sets and Systems, 117 (2001), 209-213.

[8] F. Feng, Y.B. Jun, X. Zho, Soft semirings, Computers and Mathematics with Applications, 56 (2008), 2621-2628.

[9] B. Ganji Saffar, Fuzzy n-fold obstinate and maximal (pre)filters of EQ-algebras, Journal of Algebraic Hyperstructures and Logical Algebras, 2(1) (2021), 83-98.

[10] Y. Jiang, Y. Tang, Q. Chen, An adjustable approach to intuitionistic fuzzy soft sets based decision making, Applied Mathematical Modelling, 35 (2011), 824-836.

[11] Y.B. Jun, Intuitionistic fuzzy finite switchboard state machines, Journal of Applied Mathematics and Computing, 20(1-2) (2006), 315-325.

[12] Y.B. Jun, Soft BCK/BCI-algebras, Computers and Mathematics with Applications, 56 (2008), 14081413.

[13] Y.B. Jun, Multipolar fuzzy hyper BCK-ideals of hyper BCK-algebras, Journal of Algebraic Hyperstructures and Logical Algebras, 1(1) (2020), 37-47.

[14] Y.B. Jun, K.H. Kim, Intuitionistic fuzzy ideals of BCK-algebras, International Journal of Mathematics and Mathematical Sciences, 24(12) (2000), 839-849.

[15] Y.B. Jun, K.J. Lee, J. Zhan, Soft p-ideals of soft BCI-algebras, Computers and Mathematics with Applications, 58 (2009), 2060-2068.

[16] Y.B. Jun, C.H. Park, Applications of soft sets in ideal theory of BCK/BCI-algebras, Information Sciences, 178 (2008), 2466-2475.

[17] Y.B. Jun, S.Z. Song, E.H. Roh, Soju structures with applications in BCK/BCI-algebras, Applied Mathematics - A Journal of Chinese Universities, (submitted).

[18] P.K. Maji, A.R. Roy, R. Biswas, An application of soft sets in a decision making problem, Computers and Mathematics with Applications, 44 (2002), 1077-1083.

[19] J. Meng, Y.B. Jun, BCK-algebras, Kyungmoonsa Co. Seoul, Korea 1994.

[20] D.A. Molodtsov, Soft set theory - First results, Computers and Mathematics with Applications, 37(4) (1999), 19-31, doi:10.1016/S0898-1221(99)00056-5.

[21] S.Z. Song, H.S. Kim, Y.B. Jun, Commutative ideals of BCK-algebras and BCI-algebras based on soju structures, AIMS Mathematics, (submitted).

[22] A.K. Srivastava, S.P. Tiwari, IF-topologies and IF-automata, Soft Computing, 14 (2010), 571-578.

[23] M. Touqeer, N. Cagman, On some properties of p-ideals based on intuitionistic fuzzy sets, Cogent Mathematics, 3 (2016), 1210001, doi.org/10.1080/23311835.2016.1210001.

[24] X.L. Xin, R.A. Borzooei, Y.B. Jun, Positive implicative soju ideals in BCK-algebras, Bulletin of the Section of Logic, 48(1) (2019), 1-18, doi.org/10.18778/0138-0680.48.1.01.

[25] Z. Zhang, A rough set approach to intuitionistic fuzzy soft set based decision making, Applied Mathematical Modelling, 36 (2012), 4605-4633. 